University of Maryland Francis King Carey School of Law

DigitalCommons@UM Carey Law

10-1-1997

\title{
Cancer Genetic Susceptibility Testing: Ethical and Policy Implications for Future Research and Clinical Practice
}

\author{
Benjamin S. Wilfond \\ Karen H. Rothenberg \\ University of Maryland School of Law, krothenberg@law.umaryland.edu \\ Elizabeth J. Thomson \\ Caryn Lerman
}

Follow this and additional works at: https://digitalcommons.law.umaryland.edu/fac_pubs

Part of the Genetics Commons, and the Health Law and Policy Commons

\section{Digital Commons Citation}

25 Journal of Law, Medicine \& Ethics 243 (1997).

This Article is brought to you for free and open access by the Francis King Carey School of Law Faculty at DigitalCommons@UM Carey Law. It has been accepted for inclusion in Faculty Scholarship by an authorized administrator of DigitalCommons@UM Carey Law. For more information, please contact smccarty@law.umaryland.edu. 


\section{Cancer Genetic Susceptibility Testing: Ethical and Policy Implications for Future Research and Clinical Practice}

\author{
Benjamin S. Wilfond, Karen H. Rothenberg, \\ Elizabeth J. Thomson, Caryn Lerman, for \\ the Cancer Genetic Studies Consortium, \\ National Institutes of Health
}

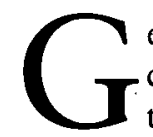
enetic testing for cancer susceptibility is an application of biotechnology that has the potential both to improve the psychosocial and physical wellbeing of the population and to cause significant psychosocial and physical harms. ${ }^{1}$ In spite of the uncertain value of genetic testing, it has captured the interest of biotechnology companies, researchers, health care providers, and the public. As more tests become feasible, pressure may increase to make the tests available and reimbursable. Both the benefits and harms of these tests lie not as much in the tests themselves, as in their power to predict or alter the future. The value of the tests does not derive from the information per se, but from the ability to communicate effectively the information to patients and providers, and the behavioral responses of patients, providers, and others to this information.

The recent marketing of genetic tests for breast can$\operatorname{cer}^{2}$ focuses attention on the health policy questions at the intersection of cancer detection and treatment, and genetic technologies. In both areas, health policy decisions to use new technologies increasingly rely on empirical data, but are also more responsive to public and professional sentiment. ${ }^{3}$ At times, these approaches yield conflicting policy positions. Although there may be a tendency to defer the introduction of new technologies until sufficient data about risks and benefits are available, there may also be a tendency to respond to public and professional requests to make potentially beneficial technologies available, even though they might not have been thoroughly evaluated. The evolving system of health care delivery in the United States and the uncertainty about which services ought to be paid for by a third party add to the complexity of health

Journal of Law, Medicine \& Ethics, 25 (1997): 243-51.

(C) 1997 by the American Society of Law, Medicine \& Ethics. policy decisions about genetic testing for cancer susceptibility.

One step taken to facilitate these health policy deliberations has been the funding of eleven studies in response to a 1994 Request for Applications by the National Institutes of Health (NIH) on studies of genetic testing and counseling for heritable breast, ovarian, and colon cancer risks. These projects, along with five others, have formed the NIH Cancer Genetics Studies Consortium (CGSC), which is coordinated by the Ethical, Legal and Social Implications Program (ELSI) of the National Human Genome Research Institute (NHGRI). CGSC's purpose is to provide a forum for discussion and collaboration regarding informed consent, study design, assessment instruments, laboratory techniques, and follow-up clinical recommendations.

The purpose of this article is to synthesize the ethical and health policy questions that are addressed by the CGSC projects. We then describe some of the broader health policy issues that will not be completely addressed by the projects in order to guide further research and health policy decision making. We argue that such policy decisions, although data-based, also involve substantive normative issues and, thus, that the CGSC projects are necessary, but not sufficient, to develop an informed policy about clinical recommendations regarding routine testing.

\section{The scope of the CGSC projects}

The CGSC projects address a broad range of clinical, psychosocial, and ethical issues relevant to genetic counseling for breast and ovarian cancer (BRCA1/BRCA2) and colon cancer (HNPCC) susceptibility. Most projects focus on people identified to be at increased risk of developing cancer because of their family histories. Some studies are assessing consumer knowledge and attitudes about genetic 
testing and counseling for cancer risks in order to learn about interest in testing as well as about needs and preferences for counseling. Other studies are examining the level of knowledge, the intentions, and the abilities of primary care physicians, nurses, oncologists, medical geneticists, and genetic counselors to educate and counsel people effectively. In these studies, knowledge areas include: understanding the impact of family history on cancer risk; interpretation of a positive or negative test; and understanding of the limitations of available options for cancer surveillance and management. Several projects are evaluating alternative methods for patient and provider education and counseling so to identify the optimal approaches. Information gained from such studies will be valuable in designing model protocols for patient and provider education, as well as for informed consent and genetic counseling services. ${ }^{4}$

An additional area of emphasis within CGSC involves evaluation of decision making about genetic testing and the impact of disclosure of mutation status. For the latter, key end points include comprehension of risk, psychosocial well-being, functional health status, and family functioning. These studies are also likely to provide insight into whether significant problems arise with employment or insurance for those who decide to undergo testing.

Of great interest is the impact of testing on participants' decisions about surveillance and prophylactic surgery. For example, it is possible that disclosure of BRCA1 mutation status may generate anxiety that leads women to avoid cancer screening tests. ${ }^{5}$ Analyses of health behavior outcomes are especially complex because of limited data on the efficacy of available surveillance and management options. For example, mammography may be less effective in younger women who have denser breast tissue. ${ }^{6}$ Concerns also exist that more frequent use of mammography will increase cancer risk; however, there are no data available to support this. Screening tests for ovarian cancer are lacking in sensitivity and specificity for women of all ages. ${ }^{7}$ Even the most extreme measures, such as prophylactic mastectomy and oophorectomy, do not eliminate risk entirely. ${ }^{8}$ CGSC investigators have developed consensus documents to provide interim guidance for follow-up care for those individuals found to have BRCA1, BRCA2, or HNPCC mutations. ${ }^{9}$ However, more data about the optimal preventive and treatment strategies are needed before more informed recommendations about genetic testing follow-up can be developed.

\section{Ethical issues highlighted by the CGSC projects}

As the CGSC projects have progressed, a number of ethical issues have been encountered. These include issues of informed consent, privacy, and confidentiality, familial implications, requests for testing children, and reproductive issues. Although these issues are not unique to genetic test- ing for cancer susceptibility, some characteristics of cancer, including the seriousness of the disorders, the undefined penetrance of mutations in the general population, and ambiguities about effective interventions, increase the complexity of these issues. The experiences of the CGSC projects with these issues provide a framework for considering similar issues involved in the clinical delivery of genetic testing services.

\section{Informed consent}

Informed consent is not just the event of signing a document; it is also a process. However, the document does represent the esșential content of the process. The documents of the consortium projects were reviewed by outside consultants and discussed at the first consortium meeting. Consortium members generally agreed that certain points should be included in all documents. First, the voluntary and optional nature of testing should be emphasized. Second, participants should be told about the limitations of testing as well as the potential benefits. These limitations include the accuracy of current diagnostic technologies, the fact that test results cannot provide definitive information about whether or when cancer will develop (for example, incomplete penetrance), and limitations in available options for prevention and surveillance. Discussion of risks should include the potential for anxiety, altered family relationships, stigmatization, and discrimination. ${ }^{10}$ But, characterizing these social risks, qualitatively and quantitatively, in a consent process is challenging.

The impact of the consent process on decision making is not clear. ${ }^{11}$ In a randomized trial of pretest education approaches among women with a family history of cancer, individualized face-to-face education and counseling did not alter testing plans. ${ }^{12}$ However, other CGSC observations indicate that motivation to have genetic testing may decrease as people receive more education. ${ }^{13}$ The goal of the process of informed consent is to allow people to make medical decisions about what they want, based on adequate understanding of relevant facts, as well as on their own values. Although surrogate markers such as comprehension and understanding or behavioral outcomes are very important, the adequacy of informed consent should be assessed from the perspective of the participants themselves. There is limited data about the appropriate content and methods for informed consent for genetic testing. These studies will provide some of this data. In the interim, CGSC established the Task Force on Informed Consent to consider the a priori components of consent for cancer genetic susceptibility testing. ${ }^{14}$

\section{Privacy and confidentiality}

CGSC members acknowledged that even though informa- 
tion about cancer risk would be kept confidential by the investigators, the potential health implications of this information increases the probability that participants would discuss these issues with their physicians. For example, the disclosure of the presence of a BRCA1 mutation to a physician may be necessary for obtaining earlier or more frequent mammograms. There was consensus that participants should be informed that such discussions may result in information being recorded in their medical records and that such recording would increase the chances of third parties gaining access to test results. Additionally, even if the information is not placed in medical records, in most states, insurers or others are not prohibited from requesting this information directly from individuals. ${ }^{15}$ The privacy and confidentiality issues are further complicated by consideration of the familial implications discussed below.

\section{Familial implications}

The identification of a cancer susceptibility mutation has a potential impact on family relationships. Further, the familial issues complicate informed consent and follow-up counseling, as well as privacy and confidentiality. This issue was faced by the investigators who wanted to recruit family members who were at increased risk to have mutations because their relatives had identified mutations. Although this issue has been acknowledged in previous genetic testing programs that focused on reproductive decision making, ${ }^{16}$ it has greater significance in genetics for susceptibility to adult-onset diseases. If general population testing should occur in the future, this will become more of an issue because every new person identified will also likely have other family members who are at increased risk.

Participants need to consider whether to inform other family members of their own risk, because this information has implications for them as well. Disclosing such information may be a means of securing social support, but it can also increase the risk of breaches of confidentiality. Some participants may decide that they do not want their relatives or friends to know their test results or may choose not to be tested because of fear of disclosure. ${ }^{17}$ Thus, counseling about this issue should occur prior to testing. There was consensus among CGSC investigators that the potential value of the information to relatives is sufficient to encourage participants to inform relatives in most circumstances. However, in some instances, the presence of complex familial relationship could lead a physician to recommend that information not be shared.

When participants want to disclose this information to family members, they may be uncertain about how to proceed. Thus, one important part of counseling may be to discuss possible strategies for effective communication with family members about test results. It may be useful to develop pamphlets or letters that give suggestions to par- ticipants about how to communicate this information, as well as to provide specific pamphlets or letters that could be given to relatives.

Nevertheless, the discussion of test results with other family members might not always be well received. In one testing strategy, generations within a family are tested in a hierarchical manner, so the result in a member of an older generation would clarify the need for testing the younger generation. Because some relatives may feel that their privacy has been violated if their names are given to investigators, it may be better for the initial contact to come from the family member. The other family members may not be aware of their increased personal risk, or may not want to know. Although not informing such individuals protects the interests of those who wish not to know, it may be at the expense of those who want to be tested. Additionally, informing an individual that he/she has a family history of a gene mutation is not the same as having a genetic test. The family member still has an opportunity to decide whether to pursue further testing to evaluate his/her personal risk.

In the protocols that are not hierarchical, a different problem emerges. Adults might want to be tested for cancer susceptibilities for which their parents have not been tested, because the parents did not want to know their status. In this case, a positive test in the adult son/daughter would imply that the parent is also positive. Although efforts could be made not to inform the parent, this may be difficult. This information might inadvertently be shared with the parent or even with third parties, including insurance companies. Although it may be tempting to err on the side of respecting the wishes of the parent and not to test their children, this would allow the parent to overrule the personal decision of the adult child. There is no legal and medical precedent for requiring parental permission in order for competent adults to make health care decisions. If such a situation were to arise, it would be desirable to engage in detailed conversations with all parties, if possible. As with many issues in clinical ethics, nonconfrontational exploration of concerns with the involved parties can lead to satisfactory compromise and resolution of the conflict. ${ }^{18}$ The CGSC studies may provide data on the conflicts between adults and their parents about testing.

Another approach to identifying research participants would be subsequent to the testing of surgical pathology tissue samples, without prior consent. Although none of the CGSC projects uses this approach, the familial implications are even greater, inasmuch as the patient does not have the opportunity to consider these issues prior to receiving the results. Even though these issues can be addressed by prior consent, it is also possible to test pathological specimens from deceased patients. In this setting, it is less clear who has authority to consent or how disagreements between family members should be resolved. ${ }^{19}$ 


\section{Requests for testing of children}

A consensus is emerging that genetic testing for adult-onset diseases should generally be deferred until adulthood. ${ }^{20}$ Children cannot provide consent, so they must rely on surrogate decision-makers. Children may be uniquely vulnerable to the psychosocial risks of testing because they are undergoing the developmental processes of acquiring a selfimage and independence. The central issue is whether the potential benefits of testing outweigh the potential harms. Although parents are usually in the best position to make such determinations, there are little data about the impact of such information on children and their families.

In the CGSC projects, children have been excluded from testing. However, individuals who are found to have mutations may still request testing for their children. Preliminary reports suggest that one of the reasons people cite for requesting genetic tests for themselves is to determine whether they could have transmitted a gene to their children. ${ }^{21}$ It is possible that some parents may also want to have their children tested. The potential benefits and risks should be discussed with participants who express such an interest, but it is appropriate to defer clinical testing in children until these benefits and risks are more clearly defined. The CGSC projects may gather data on the amount of interest expressed by parents in having their children tested. Further research is needed to delineate better the benefits and risks of such testing in children. ${ }^{22}$

\section{Reproductive issues}

In rare instances, individuals who are found to have a cancer-associated mutation may express interest in prenatal testing for cancer risks. Parents may not want to have a child with the same disease that they could develop. This raises fundamental questions regarding the appropriateness of prenatal diagnosis and pregnancy termination in such cases. ${ }^{23}$ Given the lack of information about the impact of such testing on adults, it is premature even to consider prenatal testing for cancer risks. However, it will be important to document any interest in prenatal testing for cancer risks during the course of the CGSC projects. Further, the studies may collect data about the impact of testing on other reproductive decision-making issues.

\section{Health policy implications of the CGSC projects}

\section{The clinical utility of genetic testing}

One of the primary goals of the CGSC projects is to help inform policy decisions about such testing in high-risk populations and, to a lesser extent, in the general population. The studies will provide data on the psychosocial safety and efficacy of testing. However, a number of methodological issues will create difficulties in drawing inferences from these studies to health policy decisions for routine testing in the general population.

One significant difference between these studies and routine testing is that most of the CGSC projects primarily involve high-risk families. Findings in these studies may not be applicable to the population at large, where the majority of families are at low risk. The psychosocial benefits and harms may be quite different for individuals who do not consider themselves to be at high risk or who have had no prior experience with the disease.

A second high-risk group that will emerge during these studies are relatives of those identified with mutations. In contrast to people self-identified as members of high-risk families, this second group is less likely to have had previous personal experience with cancer and less likely to have perceived themselves to be at high risk. Such individuals may have less interest in testing and may have different attitudes and behaviors in response to test results.

Furthermore, even among high-risk families, the study participants may not be representative of this population. Participants who have the motivation to enroll in the studies may be a subset of people who have different attitudes and behavioral responses than high-risk people who do not participate. Moreover, the highly structured nature of the counseling and educational interventions may yield results that are different when compared with those from the less structured approach that is likely to be provided in routine practice. Finally, the evaluative assessments that are part of the study may have an impact on participants' attitudes or behaviors about cancer genetic testing.

Just as with pharmaceutical testing, where phase I and II trials must show safety and efficacy and phase III trials demonstrate clinical utility, the data from these initial cancer studies are necessary but insufficient to show clinical utility. ${ }^{24}$ Nevertheless, the CGSC studies will lay the foundation for examination of clinical utility, which will be necessary to make policy decisions about cancer genetic testing. These studies might have to be conducted in a more routine setting, such as a primary care physician's office. Whereas research settings may provide more extensive counseling and support services, these services may be impractical in daily routine because of constraints on time, personnel, training, and reimbursement. Although studies in routine clinical settings could expose participants to some risk, the data gathered would be important in deciding whether such routine testing is safe and effective.

\section{Routine testing in a managed care environment}

Currently, decisions about reimbursement are generally made arbitrarily by third-party payers. ${ }^{25}$ There are few data about how payers will perceive genetic testing for cancer risks. Managed care organizations (MCOs) are likely to be interested in genetic testing programs that result in cost 
savings or that have greater cost-effectiveness ratios. It is also possible that they will not provide the intensive education and counseling associated with testing. In other words, MCOs may fund the test itself, but may be less likely to fund the counseling that should accompany such testing. MCOs may also be reluctant to refer patients for psychological support and genetic counseling. Although physicians could be trained and given the time to counsel patients, it is also possible that they will be expected only to provide testing, with neither adequate training nor counseling time. These concerns are speculative, but they point to a potential that the benefits of testing, as determined in research studies, may not accrue in a managed care environment. ${ }^{26}$ Thus, studies of the provision of these services within the context of managed care constraints may be helpful.

Another influence on clinical practice is marketing strategies by commercial laboratories that may promote testing to practitioners or directly to patients. Although most professional statements issued suggest that cancer genetic testing should be limited to institutional review board (IRB) approved, hypothesis-driven programs ${ }^{27}$ one statement acknowledges that clinical testing may occur outside the research setting, ${ }^{28}$ and some commercial laboratories are encouraging clinicians to provide testing more broadly. ${ }^{29} \mathrm{It}$ is possible that MCOs will require stronger evidence of benefit prior before deciding to fund testing and counseling. As long as the testing cost is greater than $\$ 1,000$ per test, a lack of third-party payment may slow the initial diffusion of genetic testing for breast cancer. However, this may only be a temporary situation, because the costs are expected to decline.

Nevertheless, the tension between commercial interests in promoting testing and managed care interests in minimizing costs point to a broader issue. Although policy decisions might be determined by the strength of these competing interests, ideally, decisions about third-party funding should follow from standard-of-care determinations. The decision about whether the benefits of testing outweigh the cost, while based on data, is sufficiently subjective to count as a social decision. Such public health decisions should be made with input from groups with other than strong financial interests. ${ }^{30}$

\section{Cost-utility data and normative evaluations}

It is possible that genetic testing for cancer susceptibility will save direct medical costs because of reduced morbidity and mortality. ${ }^{31}$ However, this may take years to establish definitively and, in fact, might not be found to be the case. The lack of evidence of cost savings, however, does not mean that such services are not a worthwhile use of resources, because saving lives usually costs money. One approach to assessing explicitly the impact of medical re- sources on society is cost-utility analysis (CUA).$^{32}$ CUA involves a determination of some combination of direct and indirect medical and nonmedical costs. These costs are then compared with some nonmonetary utility, such as measures of well-being, reduction of symptoms, quality-adjusted life years, and so forth. Cost-utility analyses and their interpretations have a large normative component that does not marginalize their importance; rather, it emphasizes the complexity of such evaluations.

One normative aspect of CUA is determining which utilities are the appropriate outcomes measures. For cancer susceptibility genetic testing, candidate measures could include decreased incidence of cancer, decreased morbidity and mortality from cancer, increased early cancer detection, improved cancer surveillance and preventive behaviors, improved well-being and quality of life, and individual satisfaction. However, outcomes can be valued differently. For example, some may consider improved wellbeing and quality of life to be a sufficient benefit, while others may argue that there should be a demonstration of decreased morbidity and mortality in order for society to invest the same resources.

Even if some agreement is reached about which outcomes should be valued, there could still be differing assessments of whether the benefits are worth the cost. People who are at high risk, insurers, and society may each be willing to spend different amounts of money to achieve the same goal. There is no defined mechanism for soliciting broad input or particular process to decide such issues. This is not a unique problem for cancer genetic testing, but one that must be faced by the health care system as a whole. However, the frenetic pace of genetic discoveries and the potential for psychosocial harms has illuminated the present need to develop a national mechanism for genetics policy development.

Similar suggestions have been made previously, ${ }^{33}$ but recently more explicit recommendations were made in a report by Mark Rothstein et al. to NIH and to the Department of Energy regarding the Human Genome Project ${ }^{34}$ and in a report by the ELSI Task Force on Genetic Testing. ${ }^{35}$ The Rothstein report proposes a federally chartered Advisory Committee on Genetics and Public Policy at the level of the secretary of the Department of Health and Human Services (DHHS). This committee would encourage participation of the public, professional societies, government regulatory agencies, and the research community in addressing issues of genetics policy and practice, legislative and regulatory policy, professional education, public education, access to and quality of genetic services, privacy and confidentiality, and discrimination based on genetic information. The committee would consist of fifteen to eighteen members with broad expertise and would establish task forces, as needed, to address emerging issues. ${ }^{36}$

The ELSI Task Force on Genetic Testing has made a 
similar recommendation that the secretary of DHHS create an Advisory Committee on Genetic Testing that would also include participation of all stakeholders. This committee would have the following goals:

[that] (a) the introduction of new genetic tests into clinical use [be] based on evidence of their analytical and clinical validity, and utility to those tested; (b) all stages of the genetic testing process in clinical laboratories meet quality standards; (c) health providers who offer and order genetic tests have sufficient competence in genetics and genetic testing to protect the well-being of their patients; and (d) there be continued and expanded availability of tests for rare genetic diseases. ${ }^{37}$

Such committees have the potential to allow the clinical integration of genetic testing into the clinical practice of medicine in a deliberative manner. They both call for decision making by relevant stakeholders, and acknowledge that decisions about clinical utility include normative deliberations. Whether such committees achieve their goals will depend on the willingness of their members to confront the ethically volatile issues involving prioritization of genetic services and to reach consensus. Additionally, at the state level, health departments could play a more active role in obtaining additional public input and in adapting national guidelines to meet local needs and conditions. One example of this approach is the Advisory Committee to the Maryland Newborn Screening Program, which was designed to provide a forum for public review of state newborn screening policy decisions. ${ }^{38}$

\section{Future research directions in cancer susceptibility testing}

Although the current CGSC projects are not designed to answer all of the policy questions that must be considered prior to wider availability of cancer genetic testing, they will contribute necessary data. Given the ultimate goal of informing health policy, it is important to consider how additional data can be obtained from the existing projects or through further longitudinal study. Supplementary data will also need to be collected in future studies of clinical utility. We recommend that the following issues be addressed.

\section{Collect data on the long-term psychosocial effects of testing}

Some issues, such as altered perceptions of self, anxiety, or other attitudinal and behavioral changes, may evolve over time. For example, even if genetic testing results in initial psychosocial distress, it is possible that, over time, partici- pants would still consider the test results to be valuable in structuring their lives. One long-term question is how those individuals who develop breast or colon cancer will perceive the experience of genetic testing. It is possible that those in whom an altered gene is identified will be more emotionally prepared when they are diagnosed with cancer. By contrast, those whose tests do not show an altered gene may feel misled. Additionally, data about long-term adverse effects that might be attributable to genetic testing (for example, loss of insurance or employment) will be important in determining what additional privacy and discrimination legislation is necessary.

\section{Measure the long-term physical effects of testing}

Even though data on the long-term physical effects of testing can be obtained from retrospective outcomes studies, it would be valuable to develop mechanisms to encourage participants in genetic testing studies to consider prospective enrollment in longer term studies of prevention, surveillance, and treatment. In anticipation that such studies will soon be implemented, the consent process for current studies should ask permission for other investigators to contact participants to inform them of other studies that become available. This would represent a unique dimension of the informed consent process, because the subject's participation is usually kept from third parties. However, safeguards to ensure that third-party requests have legitimate purposes could be provided by requiring preliminary review by the NHGRI ELSI program in addition to the usual review by IRBs.

\section{Develop a mechanism for collecting long-term information}

Individuals who believe they have experienced adverse events could provide information to a central registry, similar to the Food and Drug Administration program for reporting adverse reactions to drugs. However, a system would have to be developed for verifying adverse events. More important, problems would arise with ascertainment bias. A prospective epidemiological study of the long-term physical and psychosocial outcomes of participants who have been tested is optimal. The development of such a program would require cooperation among participants, current investigators, and funding agencies.

\section{Collect data on personnel time and approaches to consent and counseling}

An important step in understanding the costs and feasibility of genetic testing programs will be the collection of data regarding the personnel costs associated with obtaining consent and with providing counseling. Even though 
the CGSC projects are not designed to provide precise data for a rigorous cost analysis, it will be possible to make estimates of crude costs.

\section{Assess MCOs' clinical services and counseling and their effectiveness}

Research is needed to assess the willingness of MCOs to provide a range of routine clinical services and counseling, and to assess the effectiveness of these services and counseling. These data are necessary to understand whether the benefits and harms of fiscally supported research programs will occur in the routine health care setting.

\section{Research familial implications}

More conceptual and empirical research is needed about the familial implications of susceptibility testing. The CGSC projects are identifying a range of ethical and psychologi$\mathrm{cal}$ issues about the complexities of informing friends and relatives. These issues need more exploration prior to developing clinical recommendations about cancer genetic testing.

\section{Collect data on children}

Data need to be collected about participants' interests in having their children tested, and, more important, about the physical and psychosocial impact of testing children. The benefits and harms of testing in children are uncertain. As more data become available on the impact of testing on adults, that information will be important in designing studies to address these issues with respect to children.

\section{Research the implications of prenatal and preimplantation diagnosis}

Further conceptual and empirical research is needed about how genetic susceptibility testing might be used for prenatal diagnosis and preimplantation diagnosis. Even though these issues are not part of the CGSC protocols, they involve potential future uses of technologies that some people would consider valuable. Thus, more attention should be given to these issues to develop ethical and policy positions.

\section{Conclusions}

The CGSC projects are integral to policy making for genetic testing for cancer risks because they will provide important data about the safety and efficacy of such testing. Additional data is needed regarding the long-term physical and psychosocial impact of genetic testing. But, even with
CGSC studies, many policy questions about cancer testing related to clinical utility and implementation will remain unanswered: Who will provide the testing and counseling outside research settings?; How will they be trained?; Who should have access to testing and test results?; and How will testing and counseling be reimbursed? Additionally, the broader issue of the value of such programs within the context of other health services needs to be evaluated. These questions should be addressed in a systematic fashion prior to the routine utilization of such testing.

The resolution of these issues cannot be provided solely through the CGSC projects. It will require the participation of professionals, the public, and policy-makers to determine whether to expend fiscal resources to provide cancer genetic services that may improve the quality of the lives of individuals in our society.

\section{Acknowledgments}

Benjamin Wilfond, M.D., was supported by a grant from the Agency for Health Care Policy and Research (R29HS08570), and this paper was written while he was a fellow at the University of Arizona Udall Center for Studies in Public Policy. The ideas and opinions expressed in this paper are those of the authors and do not represent any position or policy of NIH, any other federal agency, or any other institution or organization to which any of the authors are affiliated. The principle investigators of CGSC are: Barbara Biesecker, M.S., National Human Genome Research Institute, Bethesda; Jeffrey Botkin, M.D., University of Utah, Salt Lake City; Deborah Bowen, Ph.D., Fred Hutchinson Cancer Research Center, Seattle; Wylie Burke, M.D., University of Washington, Seattle; Mary B. Daly, M.D., Ph.D., Fox Chase Cancer Center, Philadelphia; Bonnie Flick, M.D., University of Utah, Salt Lake City; Judy Garber, M.D., Dana-Farber Cancer Institute, Boston; Michael Green, M.D., Milton S. Hershey Medical Center, Hershey; Karen Glanz, Ph.D., University of Hawaii, Honolulu; Gail Geller, Sc.D., Johns Hopkins University, Baltimore; Ellen Gritz, Ph.D., M.D. Anderson Cancer Center, Houston; Don Hadley, M.S., National Human Genome Research Institute, Bethesda; Caryn Lerman, Ph.D., Lombardi Cancer Center, Washington, D.C.; Kenneth Offit, M.D., Memorial Sloan-Kettering Cancer Center, New York; Gloria Petersen, Ph.D., Johns Hopkins University, Baltimore; and Kathryn Taylor, Ph.D., Princess Margaret Hospital, Toronto.

\section{References}

1. See F.S. Collins, "BRCA1-Lots of Mutations, Lots of Dilemmas," N. Engl. J. Med., 334 (1996): 186-88; J.E. Garber and D. Schrag, "Testing for Inherited Cancer Susceptibility," JAMA, 275 (1996): 1928-29; B.B. Biesecker et al., "Genetic Counseling for Families with Inherited Susceptibility to Breast 
and Ovarian Cancer," JAMA, 269 (1993): 1970-74; M.C. King et al., "Inherited Breast and Ovarian Cancer: What are the Risks? What are the Choices?," JAMA, 269 (1993): 1975-80; C. Lerman et al., "BRCA1: Testing in Families with Hereditary Breast-Ovarian Cancer: A Prospective Study of Patient Decision Making and Outcomes," JAMA, 275 (1996): 1885-92; and R.T. Croyle et al., "Psychological Responses to BRCA1 Mutation Testing: Preliminary Findings," Health Psychology, 16 (1997): 63-72.

2. See G. Kolata, "Breaking Ranks, Lab Offer Test Assessing Cancer Risk," New York Times, Apr. 1, 1996, at A1; and Myriad Genetics, Press Release, Myriad Genetics Introduces the First Comprehensive Breast/Ovarian Cancer Susceptibility Test (Salt Lake City: Myriad Genetics, 1996) (on file with author).

3. See B.S. Wilfond and K. Nolan, "National Policy Development for the Clinical Application of Genetic Diagnostic Technologies: Lessons from Cystic Fibrosis," JAMA, 270 (1993): 1948-54.

4. See J.R. Botkin et al., "A Model Protocol for Evaluating the Behavioral and Psychological Effects of BRCA1 Testing," Journal of the National Cancer Center, 88 (1996): 872-82.

5. See K.M. Kash et al., "Psychological Distress and Surveillance Behaviors of Women with a Family History of Breast Cancer," Journal of the National Cancer Center, 84 (1992): 2430 .

6. See M. Morrow, "Identification and Management of the Woman at Increased Risk for Breast Cancer Development," Breast Cancer Research and Treatment, 31 (1994): 153-60.

7. See M.B. Daly and C. Lerman, "Ovarian Cancer Risk Counseling: A Guide for the Practitioner," Oncology, 7 (1993): 27-38.

8. See J.P. Struewing et al., "Prophylactic Oophorectomy in Inherited Breast/Ovarian Cancer Families," Journal of the $\mathrm{Na}$ tional Cancer Center Monographs, 17 (1995): 33-5; and M.E. Stefanek, "Bilateral Prophylactic Mastectomy: Issues and Concerns," Journal of the National Cancer Center Monographs, 17 (1995): 37-42.

9. See W. Burke et al., "Recommendations for Follow-Up Care of Individuals with an Inherited Predisposition to Cancer II. BRCA1 and BRCA2," JAMA, 277 (1997): 997-1003; and W. Burke et al., "Recommendations for Follow-Up Care of Individuals with an Inherited Predisposition to Cancer I. Hereditary Nonpolyposis Colon Cancer," JAMA, 277 (1997): 915-19.

For BRCA1/BRCA2 carriers, clinical breast examinations, mammography, transvaginal ultrasound with color Doppler, and CA-125 levels are recommended annually, beginning between ages twenty-five and thirty-five, for surveillance. This is expert opinion only and there are no studies that demonstrate any benefit. For HNPCC carriers, coloscopy is recommended to begin between ages twenty and twenty-five and to be repeated every one to three years. This recommendation is based on evidence from multiple time series with and without intervention. Transvaginal ultrasound or endometrial biopsies are recommended annually, beginning between ages twenty-five and thirty-five. This is expert opinion only and the benefit has not been proven.

10. See American Society of Clinical Oncology, Statement, "Genetic Testing for Cancer Susceptibility," Journal of Clinical Oncology, 14 (1996): 1730-36.

11. See, for example, B.J. McNeil et al., "On the Selection of Preferences for Alternative Therapies," N. Engl. J. Med., 306 (1982): 1259-62.

12. See C. Lerman et al., "Controlled Trial of Pretest Education Approaches to Enhance Informed Decision-Making for BRCA1 Gene Testing," Journal of the National Cancer Center, 89 (1997): 148-57.

13. See G. Geller et al., "Informed Consent and BRCA1
Testing," Nature Genetics, 11 (1995): 364.

14. See G. Geller et al., "Genetic Testing for Susceptibility to Adult-Onset Cancer: The Process and Content of Informed Consent," JAMA, 277 (1997): 1467-74.

15. See K.L. Hudson et al., "Genetic Discrimination and Health Insurance: An Urgent Need for Reform," Science, 270 (1995): 391-93.

16. See, for example, President's Commission for the Study of Ethical Problems in Medicine and Biomedical and Behavioral Research, Screening and Counseling for Genetic Conditions: The Ethical, Social, and Legal Implications of Genetic Screening, Counseling, and Education (Washington, D.C.: U.S. Government Printing Office, No. 203, 1983).

17. See J. Sorenson et al., "Proband and Parent Assistance in Identifying Relatives of Cystic Fibrosis Carrier Testing," American Journal of Medical Genetics, 63 (1996): 419-25.

18. See T.J. Key, "Ethics Committees and Family Ghosts: Case Studies," Journal of Clinical Ethics, 5 (1994): 19-22.

19. See E.G. DeRenzo et al., "Genetics and the Dead: Implications for Genetics Research with Samples from Deceased Persons," American Journal of Medical Genetics, 69 (1997): 33234.

20. See American Society of Human Genetics Board of Directors and the American College of Medical Genetics Board of Directors, "ASHG/ACMG Report Points to Consider: Ethical, Legal and Psychosocial Implications of Genetic Testing in Children and Adolescents," American Journal of Human Genetics, 57 (1995): 1233-41.

21. See C. Lerman et al., "Interest in Genetic Testing Among First-Degree Relatives of Breast Cancer Patients," American Journal of Medical Genetics, 57 (1995): 385-92.

22. See B.S. Wilfond et al., "Parental Requests for Genetic Testing: Research Regulations as a Guide for Clinical Testing" (1997) (unpublished).

23. See J.R. Botkin, "Fetal Privacy and Confidentiality," Hastings Center Report, 25, no. 5 (1995): 32-39; and J.M. Lancaster et al., “An Inevitable Dilemma: Prenatal Testing for Mutations in the BRCA1 Breast-Ovarian Cancer Susceptibility Gene," Obstetrics and Gynecology, 87 (1996): 306-09.

24 . Within the context of genetic testing, clinical utility refers to the direct and indirect psychosocial benefits and harms that occur after this information is conveyed to individuals. See N.A. Holtzman and M.S. Watson, eds., Promoting Safe and Effective Genetic Testing in the United States: Final Report of the Task Force on Genetic Testing (Bethesda: National Human Genome Research Institute, 1997): at 28-29.

25. See M.J. Mehlman et al., "Coverage of Genetic Technologies Under National Health Reform," American Journal of Human Genetics, 55 (1994): 1054-60.

26. See B.A. Bernhardt and R.E. Pyeritz, "The Economics of Clinical Genetics Services. III. Cognitive Genetics Services Are Not Self-Supporting," American Journal of Human Genetics, 44 (1989): 288-93.

27. See American Society of Human Genetics, "Statement of the American Society of Human Genetics on Genetic Testing for Breast and Ovarian Cancer Predisposition," American Journal of Human Genetics, 55 (1994): i-iv; National Action Plan on Breast Cancer, "Commentary on the ASCO Statement on Genetic Testing for Cancer Susceptibility," Journal of Clinical Oncology, 14 (1996): 1738-40; and National Advisory Council for Human Genome Research, "Statement on Use of DNA Testing for Presymptomatic Identification of Cancer Risk," JAMA, 271 (1994): 785.

28. See American Society of Clinical Oncology, supra note 10. 29. See, for example, Myriad Genetics Laboratories, Patient 
Brochure, Understanding Genetic Predisposition to Breast and Ovarian Cancer (Salt Lake City: Myriad Genetics, Jan. 1997) (on file with author).

30. See Wilfond and Nolan, supra note 3.

31. See M.L. Brown and L.G. Kessler, "The Use of Gene Tests to Detect Hereditary Predisposition to Cancer: Economic Considerations," Journal of the National Cancer Center, 87 (1995): 1131-36.

32. See Office of Technology Assessment, Identifying Health Technologies that Work: Searching for Evidence (Washington, D.C.: U.S. Government Printing Office, 1994).

33. See Wilfond and Nolan, supra note 3; and L.B. Andrews et al., eds., Assessing Genetic Risks: Implications for Health and Social Policy (Washington, D.C.: National Academy Press, 1994).

34. See M.A. Rothstein et al., Report of the Joint NIH/DOE Committee to Evaluate the Ethical, Legal, and Social Implications Program of the Human Genome Project (Bethesda: National Institute of Health/Department of Energy, 1996).

35. See Holtzman and Watson, supra note 24.

36. See Rothstein et al., supra note 34 , at 7.

37. See Holtzman and Watson, supra note 24, at 10-11.

38. See N.A. Holtzman. "Public Participation in Genetic Policy Making," in A. Milunsky and G.J. Annas, eds., Genetic and the Law II (New York: Plenum Press, 1980): 247-58. 
Copyright of Journal of Law, Medicine \& Ethics is the property of Blackwell Publishing Limited and its content may not be copied or emailed to multiple sites or posted to a listserv without the copyright holder's express written permission. However, users may print, download, or email articles for individual use. 